\title{
Efficacy and Safety Profile of Remimazolam for Sedation in Adults Undergoing Short Surgical Procedures
}

\author{
Yasuhiro Morimoto (D) \\ Department of Anesthesia, Ube Industries Central Hospital, Ube, Yamaguchi, Japan
}

Correspondence: Yasuhiro Morimoto, Department of Anesthesia, Ube Industries Central Hospital, Ube, Yamaguchi, Japan, Tel +8I-836-5I-922I, Email yasumorimo@gmail.com

\begin{abstract}
Sedation for short-term procedures is increasingly being used in clinical practice. Selection of appropriate drugs is important for effective and safe sedation; however, an ideal sedative remains unavailable. Remimazolam is a novel, ultrafast-acting benzodiazepine with a shorter duration of action than other agents in this class. It is currently expected to become a popular agent for short-term procedural sedation. Remimazolam shows higher clearance, a smaller volume of distribution, and a shorter half-life than midazolam. It showed dose-dependent sedative action, with onset of sedation within 60 s of administration. The results of clinical trials indicate that remimazolam is more useful than midazolam for short procedural sedation such as in patients who undergo colonoscopy and that its safety profile is comparable with that of midazolam. Anesthesia-induced vascular pain is lesser and reduction in blood pressure is lesser with remimazolam than with propofol. Moreover, the availability of flumazenil (a benzodiazepine antagonist) is a specific advantage of remimazolam. These characteristics and the results of clinical trials suggest that remimazolam will be a safer alternative to previous sedative drugs for sedation during the short surgical procedures. Although short-acting agents are useful, they might lead to immediate hyper-sedation. Remimazolam is a promising agent for short-term procedural sedation; however, clinicians should be mindful of the risks of this agent.
\end{abstract}

Keywords: sedation, propofol, midazolam, remimazolam

\section{Introduction}

Sedation for short-term procedures is increasingly being used in clinical practice. Selection of appropriate drugs is important for effective and safe sedation; however, an ideal sedative remains unavailable.

Remimazolam, a novel, ultra-short-acting intravenous anesthetic belongs to the benzodiazepine class of drugs and was first approved in Japan in 2020 as a general anesthetic. ${ }^{1}$ It was later approved in the United States in 2021 for sedation during endoscopy and has since been approved in other countries. Owing to its short duration of action and the availability of the antagonist flumazenil, it is currently expected to become a popular agent for short-term procedural sedation. Few clinical trials have discussed this subject, and currently limited data are available regarding remimazolam. In this review, I will summarize the role of remimazolam as a potential agent for short procedural sedation.

\section{Characteristics}

Remimazolam is a novel, ultrafast-acting benzodiazepine with a shorter duration of action than other agents in this class. Its pharmacological action is similar to that of midazolam. Remimazolam promotes binding of gamma-aminobutyric acid (an inhibitory neurotransmitter) to its receptors and inhibits cellular excitation in the reticular formation of the midbrain. It differs from midazolam with regard to its metabolic pathway; midazolam is metabolized via cytochrome P450 and remimazolam by hepatic tissue esterases. Additionally, alpha-hydroxymidazolam, a metabolite of midazolam, shows 1/ 8th of the sedative effect of midazolam, whereas CNS7054, a metabolite of remimazolam, shows 1/400th of its sedative 
effect. $^{2}$ This difference in metabolic pathways and pharmacokinetic profiles of its metabolites contributes to the rapid onset of action and systemic clearance of remimazolam. Remimazolam-induced sedation is reversed by flumazenil, a benzodiazepine antagonist. In contrast to other intravenous anesthetics, such as propofol, the availability of an antagonist is an advantage associated with remimazolam administration.

\section{Pharmacokinetic Profile}

The pharmacokinetics of remimazolam are previously reported. A study that compared a single intravenous infusion of remimazolam and midazolam reported that remimazolam showed higher clearance, a smaller volume of distribution, and a shorter half-life. ${ }^{3}$ It showed dose-dependent sedative action, with onset of sedation within 60 s of administration. The sedation time of remimazolam was $8 \mathrm{~min}$ and $40 \mathrm{~min}$ at a dose of $0.075 \mathrm{mg} / \mathrm{kg}$ and $0.25 \mathrm{mg} / \mathrm{kg}$, respectively. No respiratory depression or hypotension was observed following remimazolam administration.

Pharmacokinetics of continuous intravenous infusion of remimazolam was also investigated in twenty healthy men volunteers who received the following doses: $5 \mathrm{mg} / \mathrm{min}$ for $5 \mathrm{~min}, 3 \mathrm{mg} / \mathrm{min}$ for the next $15 \mathrm{~min}$, and $1 \mathrm{mg} / \mathrm{min}$ for further $15 \mathrm{~min} .{ }^{4}$ Pharmacokinetics was best described by a three-compartment model. Remimazolam showed high clearance $(1.15 \pm 0.12 \mathrm{~L} / \mathrm{min}$, mean $\pm \mathrm{SD})$, a small steady-state volume of distribution $(35.4 \pm 4.2 \mathrm{~L})$, and a short terminal phase half-life $(70 \pm 10 \mathrm{~min})$. The context-sensitive half time (CSHT) calculated using the pharmacokinetic parameters obtained in this study was as short as $6.8 \pm 2.4 \mathrm{~min}$. The mean blood pressure decreased by $24 \%$, and the heart rate increased by $28 \%$ during the study. Spontaneous breathing was maintained throughout the study. The results of this study suggest that remimazolam was characterized with fast onset and fast recovery with mild hemodynamic side effects.

\section{Results of Clinical Trials}

Currently, no clinical study has reported short-term sedation. Therefore, the results of initial clinical trials are introduced.

A phase $\mathrm{Ib}$ study was performed in multiple doses of remimazolam in volunteers undergoing colonoscopy. The study also designed to assess reversing sedative effects with flumazenil. Healthy volunteers received intravenously administered $50 \mu \mathrm{g}$ of fentanyl and remimazolam; the initial dose of remimazolam was divided as $0.04 \mathrm{mg} / \mathrm{kg}$, $0.075 \mathrm{mg} / \mathrm{kg}$, or $0.1 \mathrm{mg} / \mathrm{kg}$. ${ }^{5}$ An additional dose of $0.04 \mathrm{mg} / \mathrm{kg}$ was added at the physician's discretion during the 30 min evaluation, and $>70 \%$ of the patients were well sedated and awake within 10 min after administration. Patients who received flumazenil were awake within $1 \mathrm{~min}$ and were not re-sedated. No serious adverse events were reported. This study showed that remimazolam is useful for sedation during colonoscopy and that its sedative effect is easily antagonized by flumazenil.

In a phase IIa study, three doses of remimazolam were compared with those of midazolam. ${ }^{6}$ Patients scheduled to undergo a diagnostic upper gastrointestinal endoscopy were randomized to receive 1 of 3 doses of remimazolam or midazolam, 825 per group. The success rates of the colonoscopy were $32 \%, 56 \%$, and $64 \%$ for the low $(0.1 \mathrm{mg} / \mathrm{kg})$, medium $(0.15 \mathrm{mg} / \mathrm{kg})$, and high $(0.2 \mathrm{mg} / \mathrm{kg})$ doses of remimazolam, respectively, and $44 \%$ for midazolam $(0.075 \mathrm{mg} / \mathrm{kg})$. The time to onset of sedation was 1.5-2.5 $\mathrm{min}$ for remimazolam vs $5 \mathrm{~min}$ for midazolam. There were no obvious differences in the safety profiles of remimazolam and midazolam. The study showed that a single administration of remimazolam $(0.1-0.2 \mathrm{mg} / \mathrm{kg}$ ) was capable of inducing rapid sedation with a quick recovery in patients undergoing upper gastrointestinal endoscopy.

A Phase III study compared an initial $5 \mathrm{mg}$ dose of remimazolam followed by an additional $2.5 \mathrm{mg}$ dose of remimazolam with a placebo for outpatient colonoscopy. ${ }^{7}$ A single midazolam dose of $1.75 \mathrm{mg}$ group was also added for comparison. There were 461 randomized patients. Endoscopy was completed in $91.3 \%, 1.7 \%$, and $25.2 \%$ of patients who received remimazolam, placebo, and midazolam, respectively. Compared with midazolam, remimazolam resulted in more rapid recovery of neuropsychiatric function and earlier discharge from the hospital. The incidence of hypoxia was $1 \%$ in both the remimazolam and midazolam groups. The results of these clinical trials indicate that remimazolam is more useful than midazolam for short procedural sedation such as in patients who undergo colonoscopy and that its safety profile is comparable with that of midazolam. These clinical trials showed that remimazolam can be used safely as a sedative during endoscopy. 


\section{Advantages of Remimazolam vs Conventional Sedatives}

Compared to midazolam, remimazolam has a shorter duration of action and therefore it better regulates sedation levels. However, owing to its short duration of action, administration of additional doses may be required based on the time of the procedure. Continuous intravenous infusion is required for prolonged sedation. Surgeons who are accustomed to midazolam administration should be mindful when using remimazolam. However, the CSHT of midazolam is significantly increased with prolonged administration. Continuous intravenous infusion of remimazolam maintains prolonged sedation and thereby scores over midazolam.

Anesthesia-induced vascular pain is lesser and reduction in blood pressure is lesser with remimazolam than with propofol. The intraoperative hypotension rate was lower in the remimazolam (22\%) than in the propofol (49\%) group in a clinical trial that investigated patients who received general anesthetics. ${ }^{8}$ Both propofol and remimazolam show nearly the same CSHT; therefore, arousal time after anesthesia is nearly the same. However, the availability of flumazenil (a benzodiazepine antagonist) is a specific advantage of remimazolam. In view of the aforementioned factors, it is reasonable to conclude that remimazolam scores over propofol.

Zhang et al compared the effects of remimazolam vs propofol administration in patients who underwent hysteroscopy. ${ }^{9}$ Remimazolam was administered at a dose of $0.2 \mathrm{mg} / \mathrm{kg}$ and maintained at a dose of $1 \mathrm{mg} / \mathrm{kg} / \mathrm{hour}$. Propofol was administered at $1.5-2 \mathrm{mg} / \mathrm{kg}$ and maintained at $3-6 \mathrm{mg} / \mathrm{kg} / \mathrm{hour}$. The authors aimed to achieve a modified observer's assessment of alertness/sedation score (MOAA/S) of $\leq 2$, and remifentanil was subsequently added to the drug regimen. Vascular pain during injection occurred in $80.5 \%$ and $2.4 \%$ of patients in the propofol and remimazolam groups, respectively. Hypoxia, bradycardia, and hypotension were less frequent in the remimazolam group, which suggests that remimazolam is a safer alternative to propofol for sedation during hysteroscopy.

\section{Effects on Electroencephalography}

Electroencephalographic (EEG) monitoring, including the bispectral index (BIS) (Medtronic, Minneapolis, MN, USA) is widely used to control sedation levels in recent times and is important for evaluation of sedation levels for decisionmaking regarding the use of sedatives administered in clinical practice.

In a study that included volunteers, the authors monitored changes in the blood levels of remimazolam, the MOAA/S score, and BIS and observed that an increase in blood remimazolam concentrations was associated with a decrease in the MOAA/S score and BIS, which suggests that the BIS is a useful indicator to determine the sedative effects of remimazolam. ${ }^{10}$

EEG changes during remimazolam-induced sedation are reported to be an increase in power in the beta wave region. ${ }^{11}$ This change can be evaluated using the $\beta$-ratio utilized for the calculation of BIS; therefore, the BIS is a useful indicator of the degree of remimazolam-induced sedation.

However, studies have reported that midazolam administration which is expected to cause EEG changes similar to those caused by remimazolam does not result in a reduction in the BIS value to $<60$ despite a decrease in blood midazolam concentrations. ${ }^{12}$ Further studies are warranted to determine whether BIS can be used to confirm deeper levels of sedation. The effects of concomitant opioid administration should also be considered in clinical settings. Therefore, further accumulation of clinical data is necessary.

\section{Practical Applications}

A phase III colonoscopy study in the United States investigated the effectiveness of remimazolam for short-term sedation. ${ }^{7}$ In this study, an initial dose of remimazolam $(5 \mathrm{mg})$ was followed by an additional dose of $2.5 \mathrm{mg}$. The dose/body weight was perhaps based on the $0.075 \mathrm{mg} / \mathrm{kg}$ dose used in the Phase I study. Therefore, an initial dose of $0.075 \mathrm{mg} / \mathrm{kg}$ or $5 \mathrm{mg}$ should be administered and $50 \%$ of the dose should be added if necessary. Figure 1 shows the simulation of the concentration after the administration of an initial dose of $0.075 \mathrm{mg} / \mathrm{kg}$ followed by an additional dose of $0.0325 \mathrm{mg} / \mathrm{kg}$ at 5 and $10 \mathrm{~min}$. Simulation of intravenous anesthetics involves the blood concentration and effect-site concentration; the concentration in the central nervous system or the effect-site concentration correlates with the degree of drug-induced sedation. Pharmacokinetic simulation of remimazolam was performed using Microsoft Excel for Mac by the pharmacokinetic parameters by initial clinical trial ${ }^{13}$ (Appendix). 


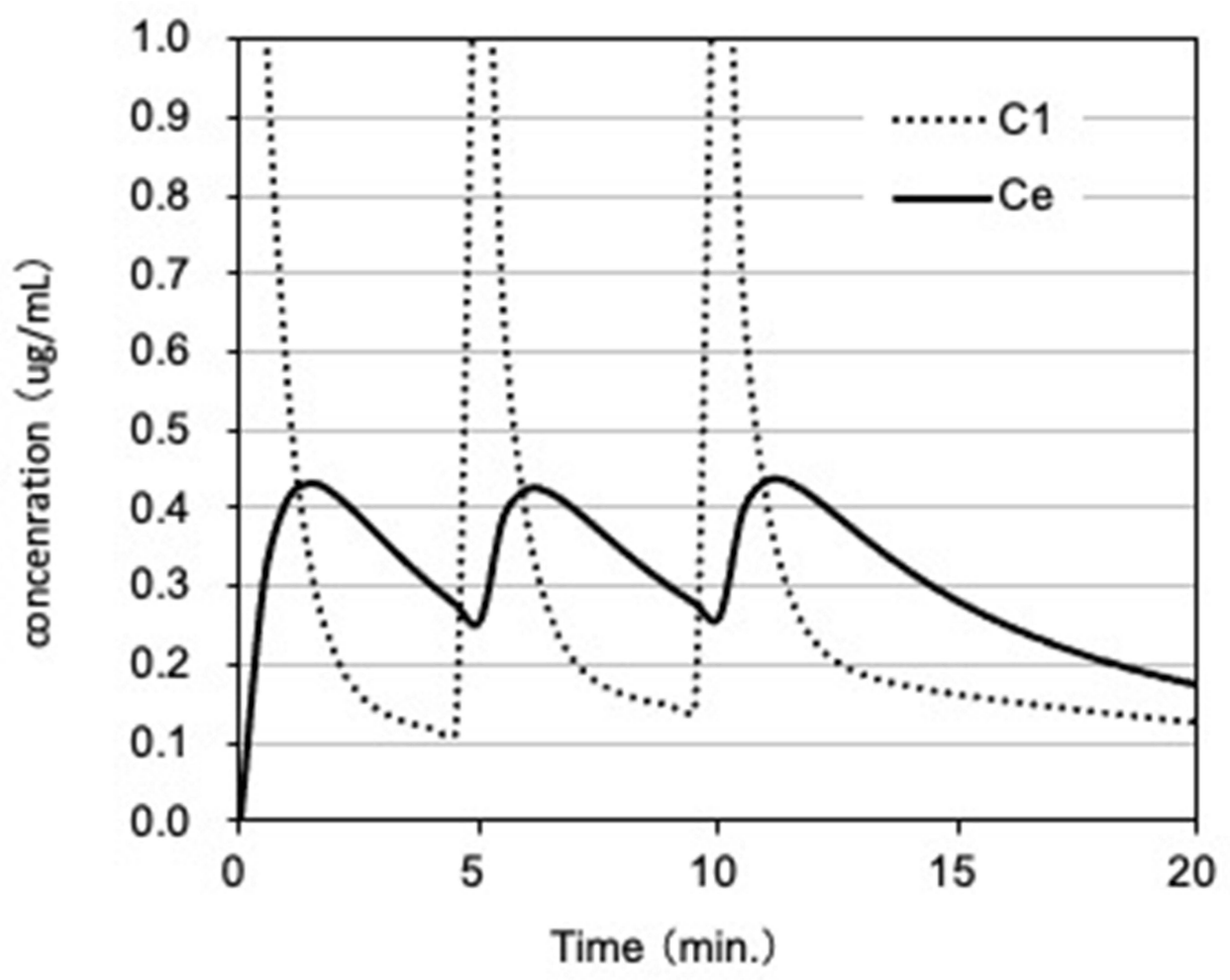

Figure I The concentration simulation after bolus administration of remimazolam. Initial dose of $0.075 \mathrm{mg} / \mathrm{kg}$ followed by an additional dose of $0.0325 \mathrm{mg} / \mathrm{kg}$ at 5 and 10 min for $60 \mathrm{~kg}$ patient.

Abbreviations: $\mathrm{Cl}$, blood concentration; Ce, effect site concentration.

A rapid decrease in the concentration is observed after a single dose of remimazolam; therefore, additional doses need to be administered every $5 \mathrm{~min}$. Reportedly, the concentration of remimazolam required to reduce the MOAA/S to $<1$ is $0.7 \mu \mathrm{g} / \mathrm{mL},{ }^{4}$ which is slightly lower than the maximum concentration of $0.45 \mu \mathrm{g} / \mathrm{mL}$. This protocol may be sufficient for sedation during short procedures, such as colonoscopy and upper gastrointestinal endoscopy. Analgesics such as fentanyl may be combined with the anesthetic, if needed.

Continuous intravenous infusion should be used for prolonged sedation. Zhang et al administered $0.2 \mathrm{mg} / \mathrm{kg}$ of remimazolam for uterine evaluation, followed by a maintenance dose of $1 \mathrm{mg} / \mathrm{kg} / \mathrm{hour}$, which is equivalent to the dose used during general anesthesia. ${ }^{9}$ It is reasonable to conclude that a lower dose can be used for sedation. Figure 2 shows the change in concentration following administration of $0.075 \mathrm{mg} / \mathrm{kg}$ as a single dose, followed by continuous intravenous infusion at the rate of $0.5 \mathrm{mg} / \mathrm{kg} /$ hour ( $50 \%$ of the dose used by Zhang et al). Nearly the same concentration was maintained after a single dose. An additional dose of $0.0325 \mathrm{mg} / \mathrm{kg}$ may be useful in patients in whom sedation appears to be insufficient.

With regard to continuous intravenous infusion, it is important to remember that the CSHT of remimazolam is approximately $7 \mathrm{~min}$. Additionally, the CSHT for the effect-site concentrations is $10 \mathrm{~min}^{4}$ If the maintenance concentration is within twice the waking concentration, the patient will be aroused $10 \mathrm{~min}$ after completion of drug administration.

Other anesthetics, such as propofol and remifentanil use target-controlled infusion (TCI), in which a pump controls the dose to maintain the desired blood concentration using pharmacokinetic parameters. TCI may likely be used for remimazolam in the near future.

\section{Complications}

A case of remimazolam-induced anaphylactic shock was reported. ${ }^{14}$ In the case, the patient may have been sensitized by previously administered midazolam. Remimazolam should not be used in patients with suspected allergy to midazolam. 


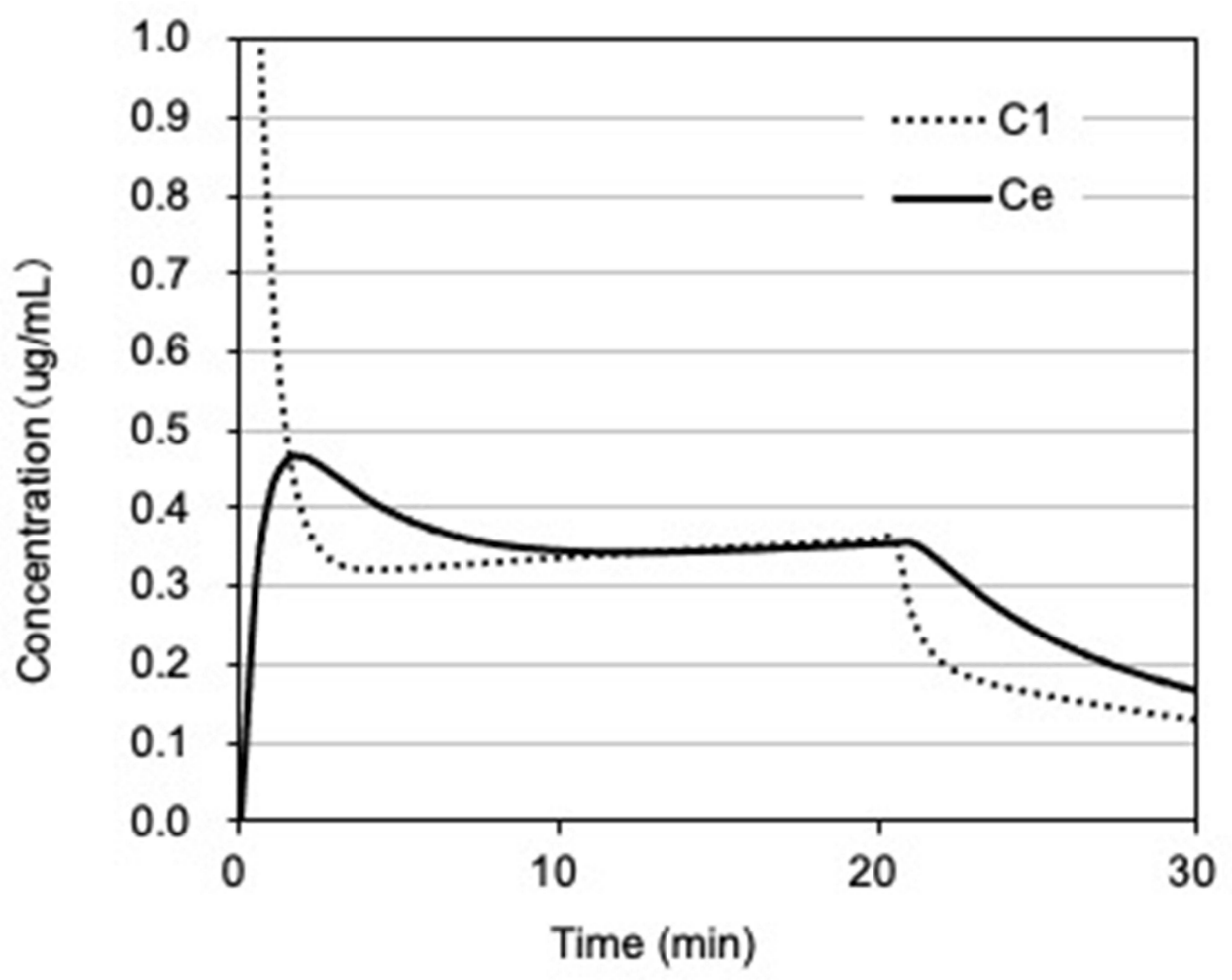

Figure 2 The concentration simulation after bolus and continuous administration of remimazolam. Administration of $0.075 \mathrm{mg} / \mathrm{kg}$ as a single dose, followed by continuous intravenous infusion at the rate of $0.5 \mathrm{mg} / \mathrm{kg} /$ hour for $60 \mathrm{~kg}$ patient.

The administration of flumazenil (a benzodiazepine antagonist) should be considered in patients with inadequate or delayed arousal. However, a re-sedation case in a patient who was aroused following flumazenil administration was reported. ${ }^{15}$ Re-sedation is likely to occur following the administration of a large volume of remimazolam during general anesthesia. However, even brief sedation can result in re-sedation. The patient should be monitored for some time after arousal by flumazenil. Further studies are warranted to confirm the optimal timing and dosage of flumazenil.

I have used remimazolam in my patients for approximately a year and have observed that its effect significantly varies between individuals. Elderly patients seem more vulnerable to the effects of remimazolam; therefore, this drug should be used cautiously in this patient population. The patient's consciousness level and respiratory status require close monitoring, and prompt respiratory support should be available if needed. Moreover, immediate administration of flumazenil should be performed to antagonize the effects of remimazolam in cases of a suspected overdose.

Although short-acting agents are useful, they might lead to immediate hyper-sedation, which may precipitate respiratory arrest and hypotension. Remimazolam is a promising agent for short-term procedural sedation; however, clinicians should be mindful of the risks of this agent and be familiar with the remedial measures in cases of a suspected overdose.

\section{Disclosure}

The author reports no conflicts of interest in this work.

\section{References}

1. Masui K. Remimazolam besilate, a benzodiazepine, has been approved for general anesthesia!! J Anesth. 2020;34:479-482. doi:10.1007/s00540020-02755-1 
2. Kilpatrik GJ, Mclntyre MS, Cox RF, et al. CNS 7056: a novel ultra-short-acting benzodiazepine. Anesthesiology. 2007;107:60-66. doi:10.1097/01. anes.0000267503.85085.c0

3. Antonik LJ, Goldwater DR, Kilpatrick GJ, et al. A placebo-and midazolam-controlled phase I single ascending-dose study evaluating the safety, pharmacokinetics, and pharmacodynamics of remimazolam 8CNS 7056): part I. Safety, efficacy, and basic pharmacokinetics. Anesth Analg. 2012;115:274-283. doi:10.1213/ANE.0b013e31823f0c28

4. Schuttler J, Eisenried A, Lerch M, et al. Pharmacokinetics and pharmacodynamics of remimazolam (CNS 7056) after continuous infusion in healthy male volunteers: part I. Pharmacokinetics and clinical pharmacodynamics. Anesthesiology. 2020;132(4):636-651. doi:10.1097/ ALN.0000000000003103

5. Worthington MT, Antonik LJ, Goldwater DR, et al. A phase Ib. dose-finding study of multiple doses of remimazolam (CNS 7056) in volunteers undergoing colonoscopy. Anesth Analg. 2013;117:1093-1100. doi:10.1213/ANE.0b013e3182a705ae

6. Borkett KM, Riff DS, Schwartz HI, et al. A phase IIa, randomized, double-blind study of remimazolam (CNS 7056) versus midazolam for sedation in upper gastrointestinal endoscopy. Anesth Analg. 2015;120:771-780. doi:10.1213/ANE.0000000000000548

7. Rex DK, Bhandari R, Desta T, et al. A phase III study evaluating the efficacy and safety of remimazolam (CNS 7056) compared with placebo and midazolam in patients undergoing colonoscopy. Gastrointest Endosc. 2018;88:427-437. doi:10.1016/j.gie.2018.04.2351

8. Doi M, Morita K, Takeda J, et al. Efficacy and safety of remimazolam versus propofol for general anesthesia: a multicenter, single-blind, randomized, parallel-group, phase IIb/III trial. $J$ Anesth. 2020;34:545-553.

9. Zhang X, Li S, Liu J. Efficacy and safety of remimazolam besylate versus propofol during hysteroscopy: single-centre randomized controlled trial. BMC Anesthesiol. 2021;21:156. doi:10.1186/s12871-021-01373-y

10. Eisenried A, Schuttler J, Lerch M, et al. Pharmacokinetics and pharmacodynamics of remimazolam (CNS 7056) after continuous infusion in healthy male volunteers: part II. Pharmacokinetics of electroencephalogram effects. Anesthesiology. 2020;132(4):652-666. doi:10.1097/ ALN.0000000000003102

11. Upton R, Martinez A, Grant C. A dose escalation study in sheep of the effects of the benzodiazepine and the respiratory and cardiovascular systems. Br J Pharmacol. 2008;155:52-61. doi:10.1038/bjp.2008.228

12. Miyake W, Oda Y, Ikeda Y, et al. Electroencephalographic response following midazolam-induced general anesthesia: relationship to plasma and effect-site midazolam concentrations. J Anesth. 2010;24(3):386-393. doi:10.1007/s00540-010-0907-4

13. Doi M. Remimazolam. J Jpn Soc Clin Anesth. 2014;34(7):860-866. Article in Japanese. doi:10.2199/jjsca.34.860

14. Tsurumi K, Takahashi S, Hiramoto Y, et al. Remimazolam anaphylaxis during anesthesia induction. J Anesth. 2021;35:571-575. doi:10.1007/ s00540-021-02934-8

15. Yamamoto T, Kurabe M, Kamiya Y. Re-sleeping after reversal of remimazolam by flumazenil. $J$ Anesth. 2021;35:322. doi:10.1007/s00540-02102915-x

Therapeutics and Clinical Risk Management

\section{Publish your work in this journal}

Therapeutics and Clinical Risk Management is an international, peer-reviewed journal of clinical therapeutics and risk management, focusing on concise rapid reporting of clinical studies in all therapeutic areas, outcomes, safety, and programs for the effective, safe, and sustained use of medicines. This journal is indexed on PubMed Central, CAS, EMBase, Scopus and the Elsevier Bibliographic databases. The manuscript management system is completely online and includes a very quick and fair peer-review system, which is all easy to use. Visit http://www. dovepress.com/testimonials.php to read real quotes from published authors.

Submit your manuscript here: https://www.dovepress.com/therapeutics-and-clinical-risk-management-journal 